\title{
INFLUÊNCIA DOS MÉTODOS PARA CÁLCULO DE GRAUS-DIA EM CONDIÇÕES DE AUMENTO DE TEMPERATURA PARA AS CULTURAS DE MILHO E FEIJÃO
}

\author{
NATALIA DOS SANTOS RENATO ${ }^{1}$, JOÃO BATISTA LOPES SILVA ${ }^{2}$, GILBERTO CHOHAKU \\ SEDIYAMA ${ }^{1}$, EDUARDO GUSMÃO PEREIRA ${ }^{1}$ \\ ${ }^{1}$ Universidade Federal de Viçosa, Viçosa, MG, Brasil \\ ${ }^{2}$ Universidade Federal do Piauí, Teresina, PI, Brasil
}

natalia.renato@ufv.br, silvajbl@yahoo.com.br, g.sediyama@ufv.br, egpereira@ufv.br

Recebido - Novembro de 2012 - Aceito Abril de 2013

\begin{abstract}
RESUMO
O presente estudo foi realizado com o objetivo de se avaliar diferentes métodos para o cálculo de grausdia (GD). Foram comparados quatro métodos de soma térmica, frequentemente usados no cálculo de GD: Ometto, Snyder, Dufault e método residual de Arnold. Esses métodos foram aplicados às culturas do milho e do feijão. As simulações foram feitas com as temperaturas observadas em Viçosa, MG no ano de 2011, e temperaturas simuladas (com acréscimo aleatório entre 0 a $5^{\circ} \mathrm{C}$ em cada dia do período). As respostas dos quatro métodos de cálculo dos GD foram similares para as temperaturas observadas (reais). Para as temperaturas elevadas, os métodos não apresentaram respostas similares. Fixando os graus-dia em $1.600^{\circ} \mathrm{C}$ dia para a cultura do milho e $1.300^{\circ} \mathrm{C}$ dia para o feijão, os métodos estudados reduziram de forma diferente o ciclo das culturas. As maiores reduções foram obtidas pelo método Arnold e Snyder, reduzindo igualmente o ciclo em 28 e 17 dias para as culturas do milho e do feijão, respectivamente. No método de Ometto, foram observadas as menores reduções, de 12 (milho) e 11 (feijão) dias, pois, nesse caso utilizam-se as temperaturas basais superior e inferior e considera-se uma penalização maior em dias em que a temperatura máxima ultrapassa a temperatura basal.
\end{abstract}

Palavras Chave: Soma térmica, temperatura do ar, milho, feijão.

\begin{abstract}
INFLUENCE OF METHODS FOR CALCULATION OF DEGREE-DAYS UNDER CONDITIONS OF TEMPERATURE INCREASE FOR MAIZE AND BEAN CROPS

The present study was performed in order to compare different methods for calculating degree-days. Four methods of thermal sum, often used in calculation of degree-days, were compared: Ometto, Snyder, Dufault and Arnold residual methods. These methods were applied to corn and beans crops. The simulations have been done considering the actual temperatures on Viçosa, MG, Brazil, in 2011, and simulated temperatures (random additions between 0 and $5^{\circ} \mathrm{C}$ for each day). The responses of the four methods on calculating the degree-days were similar for the observed temperatures in both studied crops. However, for the higher temperatures (simulated), the methods did not show similar responses. Fixing the degree days in $1,600{ }^{\circ} \mathrm{C}$ day for corn crop and $1,300{ }^{\circ} \mathrm{C}$ day for beans, the studied methods showed different reduction on the crop cycle. The largest reductions were obtained by Arnold and Snyder methods which have reduced equally the cycles by 28 and 17 days for corn and beans, respectively. The Ometto method resulted in the lowest reductions: 12 (corn) and 11 (beans) days, because it uses the upper and lower baseline temperatures, and it considers a greater penalty in days when the temperature exceeds the maximum baseline temperature.
\end{abstract}

Keywords: degree-day, temperature, maize, beans 


\section{INTRODUÇÃO}

Nos últimos anos, as mudanças climáticas têm sido motivo de grandes debates e $\mathrm{o}$ assunto tem gerado grande repercussão na comunidade científica internacional. Tais alterações podem estar relacionadas à variação do clima global ou regional ao longo do tempo. Segundo IPCC (Intergovernmental Panel on Climate Change), a temperatura média mundial já aumentou $0,6^{\circ} \mathrm{C}$ nos últimos cem anos e poderá aumentar entre 1,4 e $5,8^{\circ} \mathrm{C}$ até 2100 , se as emissões de gases do efeito estufa - principalmente $\mathrm{CO}_{2}$, $\mathrm{NH}_{3}$ e $\mathrm{CH}_{4}$ - permanecerem nas taxas atuais. Esse aumento da temperatura poderá ser acompanhado pela elevação do nível do mar, maior frequência e intensidade de ocorrência dos eventos extremos, como secas e inundações (IPCC, 2007).

Mais especificamente, especula-se que o aumento da temperatura do ar ocasionará redução na taxa fotossintética, elevação da respiração e transpiração, além da redução no ciclo das culturas, ocasionando perda de produção e produtividade. De uma forma geral, as condições climáticas futuras poderão gerar vários impactos na produtividade das culturas agrícolas (Luo et al. 2005; Zhang e Liu, 2005; Battisti e Naylor, 2009).

Para que possíveis efeitos supracitados sejam estudados, pesquisas com foco na modelagem de crescimento de plantas, ou culturas agrícolas, têm sido conduzidas (Richter e Semenov, 2005; Costa et al., 2009; Renato, 2009). Em todos os modelos, a questão do ciclo e desenvolvimento da cultura considerada são aspectos de suma importância, pois definem as mudanças fenológicas da planta e, consequentemente, os estádios de crescimento. Uma abordagem simples e muito usada para definir o estádio de desenvolvimento da cultura é o método conhecido como graus-dia (Arnold, 1959).

O conceito de graus-dia parte do seguinte princípio: o desenvolvimento de uma espécie vegetal está relacionado com o meio em cada fase fenológica ou no ciclo da cultura e é controlado a partir da soma térmica diária necessária para cada estádio. Os valores de soma térmica são diferentes entre as variedades de plantas (Schöffel e Volpe, 2002), como também os métodos de cálculo para esta soma térmica (Arnold, 1959; Ometto, 1981; Snyder, 1985; Dufault, 1997).

Segundo Ometto (1981), existe uma temperatura mínima para acionar os dispositivos metabólicos da planta, que é denominada de temperatura basal inferior $(\mathrm{Tb})$. Somente acima desta temperatura a planta pode se desenvolver. O mesmo autor ressalta, contudo, que a planta também possui uma temperatura basal superior (TB), acima da qual há um estancamento das atividades metabólicas, prejudicando seu desenvolvimento.

O método de Arnold (1959) considera somente a Tb, enquanto os métodos propostos por Ometto (1981), Snyder (1985) e Dufalt (1997) também consideram que as plantas possuem uma temperatura basal superior.
O método de Arnold (1959) é o mais usado por ser um método simples. Os graus-dia, neste método, são calculados como a soma da diferença entre a temperatura média diária e a temperatura basal inferior. A grande vantagem nesse caso é a simplicidade, porém, como desvantagem cita-se o fato de que este método não considera a temperatura basal superior.

Os valores de temperatura basal inferior e superior variam de acordo com a espécie e o cultivo estudados. Alguns trabalhos também utilizam valores diferentes para cada etapa do desenvolvimento da planta (Souza, 1996). Para a cultura do milho (Zea mays L.), por exemplo, normalmente é utilizado um valor de $10^{\circ} \mathrm{C}$ como temperatura basal inferior e $32^{\circ} \mathrm{C}$ como temperatura basal superior (Romano, 2005; Assis et al., 2006). Já para a cultura do feijão (Phaseolus vulgaris L.), emprega-se $10^{\circ} \mathrm{C}$ para a temperatura basal inferior e $35^{\circ} \mathrm{C}$ para a basal superior (Miranda e Campelo Júnior, 2010).

Os modelos de crescimento de plantas têm mostrado que em cenários de temperaturas muito elevadas, os ciclos das culturas diminuem, reduzindo a produtividade (Siqueira et al., 2001; Streck e Alberto, 2006; Costa et al., 2009, Renato, 2009). Ao simular a produtividade da cultura do milho e feijão, para o cenário A2 do IPCC projetado pelo HadCM3 até o ano de 2080, Costa et al. (2009) observaram uma queda na produtividade de até $39 \%$ para o feijoeiro e de $31 \%$ para a cultura do milho, sendo um dos fatores da queda a redução do ciclo dessas culturas. Mearns et al. (1997), utilizando o modelo CERES-Wheat nos EUA, simulando um aumento de $2{ }^{\circ} \mathrm{C}$ na temperatura média, encontraram resultados que reduziram a produtividade média em $25 \%$ e o ciclo para o trigo.

De uma forma geral, os modelos de simulação fazem a estimativa da produtividade das culturas da seguinte forma: calculam a fotossíntese líquida e utilizam os graus-dia acumulados para fracionar os carboidratos acumulados nos diferentes órgãos da planta. À medida que os graus-dias são acumulados, a planta passa por diferentes estádios, nos quais os coeficientes de partição também variam.

O método para o cálculo do GD tem papel importante na estimativa da produtividade estimada pelos modelos de crescimento de plantas em cenários de mudanças climáticas, pois controlam o ciclo e desenvolvimento da cultura. Nesse sentido, o presente estudo foi realizado com o objetivo de se comparar os diferentes métodos de cálculo de GD na modelagem do ciclo das culturas do milho e feijão em cenários futuros, com elevação das temperaturas.

\section{MATERIAL E MÉTODOS}

No presente estudo, foram avaliados quatro métodos de soma térmica, frequentemente, usados no cálculo de GD: método residual de Arnold (1959), método de Ometto (1981), 
método de Snyder (1985) e método de Dufault (1997).

O método proposto por Arnold (1959) considera apenas a temperatura basal inferior $(\mathrm{Tb})$ no cálculo dos graus-dia (Equação 1).

$$
\mathrm{GD}=\frac{\mathrm{TM}+\mathrm{Tm}}{2}-\mathrm{Tb}
$$

em que: $\mathrm{GD}=$ graus-dia, ${ }^{\circ} \mathrm{C} ; \mathrm{TM}=$ temperatura máxima do dia, ${ }^{\circ} \mathrm{C} ; \mathrm{Tm}=$ temperatura mínima do dia, ${ }^{\circ} \mathrm{C} ; \mathrm{Tb}=$ temperatura basal inferior, ${ }^{\circ} \mathrm{C}$.

O método proposto por Ometto (1981), além de considerar a temperatura basal inferior, considera também a temperatura basal superior (TB). Neste método a soma térmica apresenta cinco condicionantes, cada uma com determinada equação (Equações 2 a 6) para cálculo de GD:

$$
\begin{array}{ll}
\mathrm{TB}>\mathrm{TM}>\mathrm{Tm}>\mathrm{Tb} & \mathrm{GD}=\frac{\mathrm{TM}-\mathrm{Tm}}{2}+\mathrm{Tm}-\mathrm{Tb}, \\
\mathrm{TB}>\mathrm{TM}>\mathrm{Tb}>\mathrm{Tm} & \mathrm{GD}=\frac{(\mathrm{TM}-\mathrm{Tb})^{2}}{2(\mathrm{TM}-\mathrm{Tm})}, \\
\mathrm{TB}>\mathrm{Tb}>\mathrm{TM}>\mathrm{Tm} & \mathrm{GD}=0,
\end{array}
$$

$\mathrm{TM}>\mathrm{TB}>\mathrm{Tm}>\mathrm{Tb}$

$$
\mathrm{GD}=\frac{2(\mathrm{TM}-\mathrm{Tm})(\mathrm{Tm}-\mathrm{Tb})+(\mathrm{TM}-\mathrm{Tm})^{2}-(\mathrm{TM}-\mathrm{TB})}{2(\mathrm{TM}-\mathrm{Tm})},
$$

$$
\mathrm{TM}>\mathrm{TB}>\mathrm{Tb}>\mathrm{Tm} \quad \mathrm{GD}=\frac{1}{2} \cdot \frac{(\mathrm{TM}-\mathrm{Tb})^{2}-(\mathrm{TM}-\mathrm{TB})^{2}}{\mathrm{TM}-\mathrm{Tm}},
$$

em que: $\mathrm{TB}=$ temperatura basal superior $\left({ }^{\circ} \mathrm{C}\right)$

O método proposto por Snyder (1985), também, considera a temperatura basal superior e a inferior, porém apresenta somente quatro condições, com equações mais complexas (Equações 7 a 13):

$$
\begin{aligned}
& \mathrm{TB}>\mathrm{TM}>\mathrm{Tm}>\mathrm{Tb} \quad \mathrm{GD}=\frac{\mathrm{TM}+\mathrm{Tm}}{2}-\mathrm{Tb}, \\
& \mathrm{TB}>\mathrm{TM}>\mathrm{Tb}>\mathrm{Tm} \quad \mathrm{GD}=\frac{(\mathrm{M}-\mathrm{Tb})\left(\frac{\pi}{2}-\theta\right)+\mathrm{W} \cos (\theta)}{\pi}, \\
& \theta=\operatorname{arsen}\left(\frac{\mathrm{Tb}-\mathrm{M}}{(\mathrm{W})}\right), \\
& \mathrm{M}=\frac{\mathrm{TM}+\mathrm{Tm}}{2} \quad \mathrm{~W}=\frac{\mathrm{TM}-\mathrm{Tm}}{2}, \\
& \mathrm{GD}=\mathrm{M}-\mathrm{Tb}-\frac{(\mathrm{M}-\mathrm{TB})\left(\frac{\pi}{2}-\phi\right)+\mathrm{W} \cos (\phi)}{\pi}
\end{aligned}
$$

$$
\begin{aligned}
& \phi=\operatorname{arcsen}\left(\frac{\mathrm{TB}-\mathrm{M}}{\mathrm{W}}\right), \\
& \mathrm{GD}=\frac{(\mathrm{M}-\mathrm{Tb})\left(\frac{\pi}{2}-\theta\right)+\mathrm{W} \cos (\theta)}{\pi}-. \\
& \frac{(\mathrm{M}-\mathrm{TB})\left(\frac{\pi}{2}-\phi\right)+\mathrm{W} \cos (\phi)}{\pi}
\end{aligned}
$$

No método proposto por Dufault (1997) existem apenas duas condições (Equações 14 e 15). Diferentemente dos métodos de Ometto (1981) e de Snyder (1985), neste método não existe uma condição quando a temperatura mínima fica abaixo da temperatura basal inferior.

$$
\begin{aligned}
& \mathrm{TB}<\mathrm{TM} \quad \mathrm{GD}=\frac{\mathrm{TM}-\mathrm{Tm}}{2}-\mathrm{Tb}, \\
& \mathrm{TB}>\mathrm{TM} \quad \mathrm{GD}=\frac{(2 \mathrm{~TB}-\mathrm{TM}+\mathrm{Tm})}{2}-\mathrm{Tb} .
\end{aligned}
$$

Para avaliar os modelos de GD foi utilizado o software ModelMaker. Nas simulações foram considerados dois cenários: (i) temperaturas diárias observadas de janeiro de 2011 a maio de 2011 (TdOb); (ii) temperaturas diárias simuladas com acréscimo de até $5^{\circ} \mathrm{C}$ aleatoriamente nas temperaturas observadas (TdSA).

Os dados de entrada foram obtidos a partir de dados observados de temperatura máxima e mínima de Viçosa, MG $\left(20^{\circ} 45^{\prime} \mathrm{S}, 42^{\circ} 52^{\prime} \mathrm{W}\right)$, do ano de 2011 , coletados por uma estação meteorológica automática pertencente ao INMET. Como as simulações foram feitas para observar o ciclo da cultura em condições de temperatura elevada (mudanças climáticas), foi desenvolvida, adicionalmente, uma sub-rotina, que acrescentava valores de 0 a $5^{\circ} \mathrm{C}$ de forma aleatória aos valores observados da temperatura para as condições de Viçosa (Figura 1).

As temperaturas basais inferiores utilizadas no modelo para as culturas do milho e do feijão foram $10^{\circ} \mathrm{C}$. As temperaturas basais superiores foram de $32^{\circ} \mathrm{C}$ para o milho e de $35^{\circ} \mathrm{C}$ para o feijão.

As simulações foram feitas para o ciclo fixo de 130 dias para a cultura do milho e 100 dias para a cultura do feijoeiro. Também foi realizada outra simulação com os graus dias acumulados (GDA) fixo ou constantes térmicas, com 1.600 ${ }^{\circ} \mathrm{C}$ dia para a cultura do milho e $1.300^{\circ} \mathrm{C}$ dia para a cultura do feijoeiro.

\section{RESULTADOS E DISCUSSÕES}

Para a cultura do milho, que tem uma temperatura basal superior (TB) de $32^{\circ} \mathrm{C}$, observa-se que as temperaturas máximas observadas (TmaxOb) mantiveram-se inferiores ao valor da TB em $90 \%$ do tempo. Já para a cultura do feijoeiro, que tem uma 


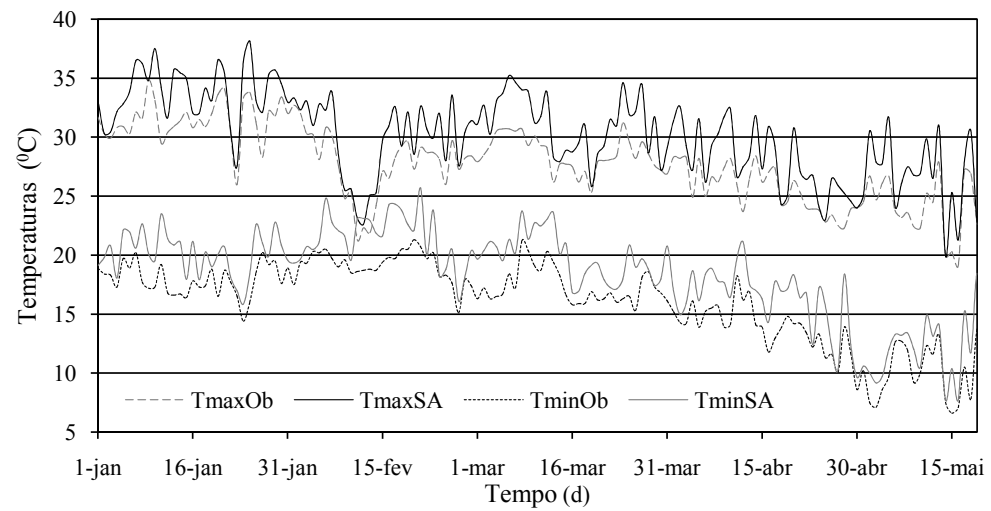

Figura 1 - Temperaturas máximas (TmaxOb) e mínimas (TminOb) de Viçosa, MG, no ano de 2011 e temperaturas máximas (TmaxSA) e mínimas (TminSA) simuladas com um aumento aleatório de até $5{ }^{\circ} \mathrm{C}$ nos valores observados de temperatura.

temperatura basal superior de $35^{\circ} \mathrm{C}$, as temperaturas observadas (TmaxOb) não ultrapassaram tal limite (Figura 1). Dessa forma, os quatro modelos responderam de forma similar para a cultura do milho (Figura 2) e para o feijoeiro (Figura 3).

As respostas dos quatro métodos de cálculo dos GD são similares para temperaturas reais sem acréscimo (Figuras 2 e 3). No caso do feijoeiro, os quatro modelos de graus-dia completaram $1.300^{\circ} \mathrm{C}$ dia em 100 dias. Já na cultura do milho o modelo completou $1.600^{\circ} \mathrm{C}$ dia em 130 dias com as equações de Arnold e Snyder, 132 dias com a equação de Dufalt, e 138 dias de acordo com a equação de Ometto. Esses resultados justificam o uso, na maioria dos modelos, da equação mais simples, proposta por Arnold, uma vez que um dos objetivos da modelagem é a representação de um sistema real da forma mais simplificada possível.

Entretanto, cabe ressaltar que em alguns trabalhos abordando o estudo das mudanças climáticas e seus impactos na agricultura, tem-se encontrado algumas dificuldades com o uso desta simplificação (Costa et al., 2009; Renato, 2009), pois os modelos de projeções atmosféricas têm apontado temperaturas muito elevadas para os cenários futuros (Mearns et al., 1997;
Renato, 2009; Costa et. al, 2009. Isto tem resultado em uma redução muito drástica no ciclo das culturas com o uso do método de GD de Arnold (1959), uma vez que não prevê uma penalização por temperaturas acima da temperatura basal superior.

Para as temperaturas elevadas (TmaxSA), os métodos de cálculo de graus-dias não convergem mais para uma resposta similar no caso da cultura do milho (Figura 4). Nos dias em que os valores de temperaturas máximas foram maiores do que a temperatura basal superior, os modelos passaram a responder de forma diferente. Para o método proposto por Arnold (1959), por exemplo, quanto maior a temperatura média do dia, maior foi o acúmulo dos graus-dia, sem considerar um limite superior para este acréscimo. Já para os outros métodos, em dias com temperaturas maiores que a temperatura basal superior, a planta é submetida a uma penalização no acúmulo dos graus-dia. No caso do feijoeiro, a diferença foi um pouco menor, uma vez que esta cultura apresenta ciclo menor e temperatura basal superior maior (Figura 5).

As simulações dos ciclos das culturas feitas com os graus-dias fixos, e temperaturas elevadas (TdSA), apresentaram redução nos ciclos de ambas as culturas (Tabela 1). Nessas

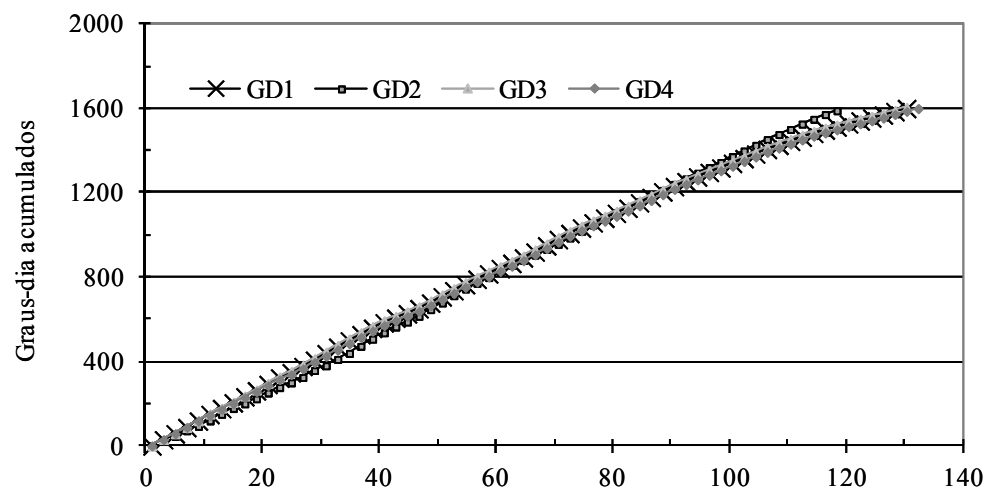

Figura 2 - Simulação dos graus-dia acumulados (GDA - TdOb) para a cultura do milho com temperaturas observadas de Viçosa, MG, em 2011, de acordo com os métodos: GD1 - Arnold (1959); GD2 - Ometto (1981); GD3 - Snyder (1985); e GD4 - Dufalt (1985). 


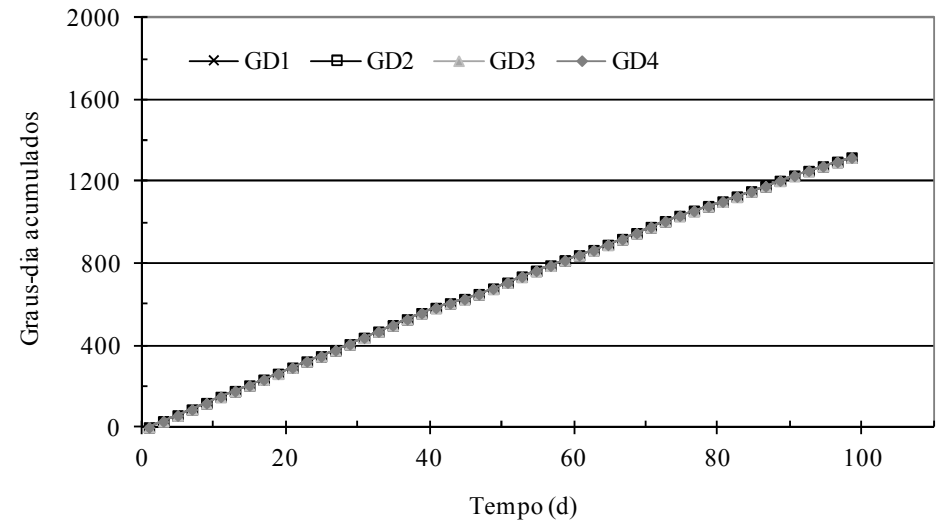

Figura 3 - Simulação dos graus-dia acumulados (GDA - TdOb) para a cultura do feijoeiro com temperaturas observadas de Viçosa, MG, em 2011, de acordo com os métodos: GD1 - Arnold (1959); GD2 - Ometto (1981); GD3 - Snyder (1985); e GD4 - Dufalt (1985).

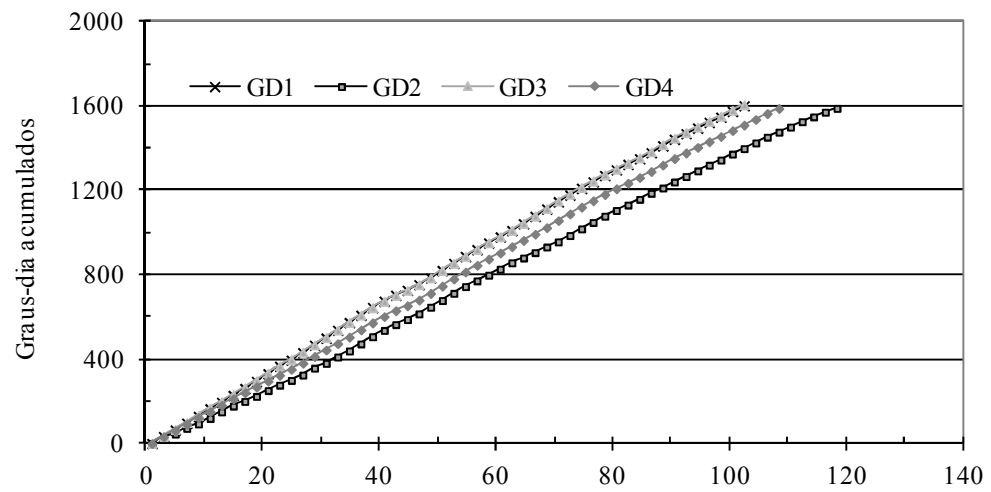

Figura 4 - Simulação do acúmulo de graus dia (GDA - TdSA) para a cultura do milho com temperaturas simuladas, de acordo com os métodos: GD1 - Arnold (1959); GD2 - Ometto (1981); GD3 - Snyder (1985); e GD4 - Dufalt (1985).

Tabela 1 - Ciclo, em dias, das culturas do milho e do feijão, com temperaturas elevadas, de acordo com os métodos: GD1 - Arnold (1959); GD2 - Ometto (1981); GD3 - Snyder (1985); e GD4 - Dufalt (1985).

\begin{tabular}{lcccc}
\hline Cultura & GD1 & GD2 & GD3 & GD4 \\
\hline Milho & 102 & 118 & 102 & 107 \\
Feijão & 83 & 89 & 83 & 83 \\
\hline
\end{tabular}

condições, os métodos considerados mostraram redução no ciclo de forma diferenciada (Tabela 2).

Considerando-se que as culturas mantiveram o seu ciclo com os mesmos graus-dias, no método de Arnold (1959) e Snyder (1985), o ciclo diminuiu 28 e 17 dias para a cultura do milho e do feijão, respectivamente, pois ambos os métodos não apresentam penalização severa ou nenhuma penalidade para as temperaturas acima da temperatura basal superior. Para o método de Dufalt (1997), que somente utiliza a temperatura basal superior, o ciclo diminui 24 e 17 dias, para as culturas de milho e feijão, respectivamente. Já no método de Ometo (1981) esta redução foi menor, 12 e 11 dias, pois tal método utiliza ambas as temperaturas basais, superior e inferior, e considera uma penalização maior em dias que a temperatura máxima ultrapassa os valores da TB.

Lima e Da Silva (2008), avaliando todos os métodos citados anteriormente, em estudos com a cultura do café em condições de temperaturas observadas, concluíram que o método de Ometto (1981) foi o que melhor caracterizou a soma térmica (Graus-dia) para as cultivares estudadas (Acácia Cerrado e Rubi), corroborando com os resultados obtidos neste trabalho. Zucareli et al. (2010) estudaram a tendência do acúmulo de graus-dia e a produtividade para diferentes genótipos do milho para a cidade de Londrina, PR e obtiveram resultados diferentes para os diversos genótipos da cultura do milho. $\mathrm{O}$ acúmulo de graus-dia para completar o ciclo foi diferente para os diferentes genótipos estudados.

Percebe-se, portanto, que o maior problema do uso dos graus-dia na modelagem da produtividade agrícola em cenários de mudanças climáticas, é que, geralmente, adota-se o método de Arnold (1959), fórmula mais simples dentre as disponíveis 


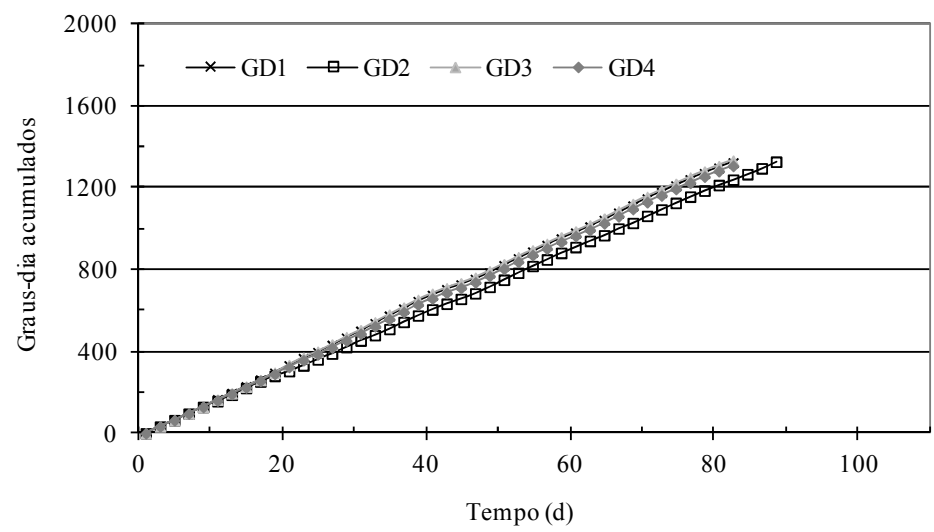

Figura 5 - Simulação do acúmulo de graus dia (GDA - TdSA) para a cultura do feijoeiro com temperaturas simuladas, de acordo com os métodos: GD1 - Arnold (1959); GD2 - Ometto (1981); GD3 - Snyder (1985); e GD4 - Dufalt (1985).

Tabela 2 - Diferença, em dias, nos ciclos das culturas do milho e do feijão quando simulados com temperaturas observadas e simuladas de acordo com os métodos de GD1 - Arnold (1959), GD2 - Ometto (1981), GD3 - Snyder (1985) e GD4 - Dufalt (1985).

\begin{tabular}{lcccc}
\hline Diferença do Ciclo (dias) & GD1 & GD2 & GD3 & GD4 \\
\hline Milho & 28 & 12 & 28 & 24 \\
Feijão & 17 & 11 & 17 & 17 \\
\hline
\end{tabular}

(Equação 1), para se calcular a soma térmica da cultura. A equação citada, no entanto, não considera as temperaturas basal superiores, uma vez que a planta, quando submetida às temperaturas elevadas, tem sua soma térmica maior. Tal procedimento tem como resultado o cômputo do tempo do ciclo da cultura de forma reduzida, o que é irreal.

O método de Snyder (1985), apesar de considerar a temperatura basal superior, melhorando as simulações para o cenário de temperaturas elevadas, apresentou resultados similares ao método de Arnold (1959). Porém, tal método é mais complexo, por exigir um número maior de cálculos.

No caso do método de Ometto (1981), o efeito citado anteriormente na redução do ciclo da cultura é reduzido, uma vez que temperaturas muito elevadas não são mais somadas linearmente no desenvolvimento do ciclo das culturas. Para a cultura do milho observa-se melhor simulação com o uso do método de Ometto (1981) em condições de temperatura elevadas, pois esta cultura possui ciclo maior e temperatura basal superior menor quando comparado com o feijoeiro.

Os resultados encontrados por Renato (2009) mostram grande diminuição no ciclo da cultura da cana-de-açúcar em cenários de mudanças climáticas, utilizando temperaturas projetadas pelo modelo HADCM3 para o ano de 2020. Os cálculos foram feitos a partir do modelo de Arnold (1959), porém este efeito seria visivelmente menor com o uso do modelo de Ometto (1981), uma vez que a cana-de-açúcar possui um ciclo longo, de aproximadamente um ano.

\section{CONCLUSÕES}

Em condições de temperaturas muito elevadas torna-se importante o uso de equações com limites inferiores e superiores para o desenvolvimento das plantas. Nas culturas estudadas, tanto para o milho quanto para o feijão, o método de Ometto (1981) é o mais indicado para as simulações, principalmente, em cenários de mudanças climáticas que projetam maiores incrementos da temperatura do ar.

\section{REFERÊNCIAS BIBLIOGRÁFICAS}

ARNOLD, C. Y. The determination and significance of the base temperature in a linear heat unit system. Proceedings of the American Society for Horticultural Science, Alexandria, v. 74, n. 1 p. 430-445, 1959.

ASSIS, J. P.; DOURADO NETO, D.; NASS, L. L.; MANFRON, P. A.; BONNECARRERE, R. A. G.; MARTIN, T. N. Simulação estocástica de atributos do clima e da produtividade potencial de milho utilizando-se distribuição triangular. Pesquisa Agropecuária Brasileira, Brasília, v. 41, p. 539-543, 2006.

BATTISTI, D. S.; NAYTOR, R. L. Historical warnings of future food insecurity with unprecedented seasonal heat. Science, Washington, v. 323, n.5911, p. 240-244, 2009.

COSTA, L. C.; JUSTINO, F.; OLIVEIRA, 1. J. C.; SEDIYAMA, G. C.; FERREIRA, W. P. M.; LEMOS, C. F. Potential forcing of $\mathrm{CO}_{2}$, technology and climate changes in maize (Zea mays) and bean (Phaseolus vulgaris) yield in southeast Brazil. Environmental Research Letters, Bristol, v. 4, n. 1, p. 1-10, 2009. 
DUFAULT, R. J. Determining heat unit requirements for broccoli in coastal South Carolina. Journal of the American Society for Horticultural Science, Alexandria, v.122, n.2, p.169-174, 1997.

IPCC (Intergovernmental Panel on Climate Change). Climate Change 2007: The Physical Science Basis. Cambridge University Press: Cambridge, 2007.

LUO, Q.; BELLOTI, W.; WILLIAMS, M.; BRYAN, B. Potential impact of climate change on wheat yield in South Australia. Agricultural and Forest Meteorology, Amsterdam, v.132, n.3-4 p. 273-285, 2005.

LIMA, E. P.; Da SILVA, E. L. Temperatura base, coeficientes de cultura e graus-dia para cafeeiro arábica em fase de implantação. Revista Brasileira de Engenharia Agrícola e Ambiental, Campina Grande, v.12, n.3, p.266-273, 2008 MEARNS, L.O., ROSENZWEIG, C., GOLDBERG, R. Mean and variance change in climate scenarios: Methods, agricultural applications, and measures of uncertainty. Climatic Change, Dordrecht, v.35, n.4, p.367-96, 1997.

MIRANDA, M. N.; CAMPELO JÚNIOR. Soma térmica para o subperíodo semeadura-maturação de feijão cv. carioca em colorado do oeste, Rondônia. Pesquisa Agropecuária Tropical, Goiânia, v. 40, n. 2, p. 180-185, 2010.

OMETTO, J. C. Bioclimatologia vegetal. São Paulo: Agronômica Ceres 1981. 440p.

RENATO, N.S. Fotossínteses em cenários de mudanças climáticas: Adaptação de modelo para a produtividade potencial da cana de açúcar. Viçosa, MG: UFV, 2009, 53f. Dissertação (Mestrado em Meteorologia Agrícola), Universidade Federal de Viçosa, Viçosa, MG, 2009.

RICHTER, G. M.; SEMENOV, M. A. Modelling impacts of climate change on wheat yields in England and Wales: assessing drought risks. Agricultural Systems, Amsterdam v. 84, p. 77-97, 2005.

ROMANO, M. R. Desempenho fisiológico da cultura de milho com plantas de arquitetura contrastante: parâmetros para modelos de crescimento. Piracicaba, SP: USP, 2005, 100f. Tese (Doutorado em Agronomia), Universidade de São Paulo, Piracicaba, SP, 2005.
SCHÖFFEL, E. R.; VOLPE, C. A. Relação entre a soma térmica efetiva e o crescimento da soja. Revista Brasileira de Agrometeorologia, Santa Maria, v. 10, n. 1, p. 89-96, 2002.

SIQUEIRA, O. J. W.; STEINMETZ, S.; SALLES, L. A. B.; FERNANDES, J. M. Efeitos potenciais das mudanças climáticas na agricultura brasileira estratégias adaptativas para algumas culturas. In: Embrapa. Mudanças climáticas globais e a agropecuária brasileira. Embrapa Meio Ambiente, Jaguariúna, SP, p. 33 - 64, 2001.

SOUZA, A. Relações entre estádios fenológicos de três cultivares de arroz e graus-dia e dias de calendário para a microrregião de Dourados - MS. Revista Brasileira de Meteorologia, São José dos Campos ,v. 1 n. 1-2, p. 18-24, 1996.

SNYDER, R. L. Hand calculating degree days. Agriculture and Forest Meteorology, Amsterdam, v. 35, n. 1-4, p.353358, 1985.

STRECK, N. A.; ALBERTO, C. M. Estudo numérico do impacto da mudança climática sobre o rendimento de trigo, soja e milho. Pesquisa Agropecuária Brasileira. Brasília: v. 41, n. 9, p.1351-1359, 2006.

ZHANG, X. C.; LIU, W. Z. Simulating potential response of hydrology, soil erosion, and crop productivity to climate change in Changwu tableland region on the Loess Plateau of China. Agricultural and Forest Meteorology, Amsterdam, v. 131, n. 3-4, p.127-142, 2005.

ZUCARELI, C.; CARMEIS FILHO, A. C. A.; GONÇALVES, M. S.; OLIVEIRA, M. Acúmulo de Graus dias, Ciclo e Produtividade de Cultivares de Milho de Segunda Safra para a Região de Londrina-PR. In: XXVIII Congresso Nacional de Milho e Sorgo, Anais... 2010. 